\title{
Computation of Wound Rotor Induction Machines Based on Coupled Finite Elements and Circuit Equation under a First Space Harmonic Approximation
}

\author{
Smail Mezani ${ }^{1,2}$, Tahar Hamiti ${ }^{1}$, Lamia Belguerras ${ }^{1}$, Thierry Lubin ${ }^{2}$, Christopher Gerada ${ }^{1}$ \\ ${ }^{1}$ The University of Nottingham, PEMC group, Nottingham, NG7 2RD, UK \\ ${ }^{2}$ Université de Lorraine, FST, Laboratoire GREEN, Vandœuvre-lès-Nancy, 54506, France
}

\begin{abstract}
The paper presents a fast method to compute wound rotor induction machines in steady state. Coupled time-harmonic FE-circuit equation are used under a first space harmonic approximation for the air-gap magnetic field. It is shown that only 4 magnetostatic FE computations are necessary to determine the machine performances for any slip value. The performances comparison to a conventional complex magnetodynamic and time stepping FE analyses show the effectiveness of the proposed approach.
\end{abstract}

Index Terms - Circuit equation, Finite element analysis, Fourier series, Induction machine, Wound rotor

\section{INTRODUCTION}

$\mathrm{W}$ OUND rotor induction machines (WRIM) are widely used when a high starting torque and low current is needed. They also constitute a good alternative drive where limited range adjustable speed is required.

The design of WRIM can be done using a variety of methods. The concepts of electric and magnetic loadings together with manufacturers past experience allows an initial sizing of the machine [1]. However, finite element (FE) analyses give the most accurate results. They allow a full transient description accounting for saturation and circuit equation coupling [2]. Unfortunately, such an analysis is very costly in terms of computation time especially when only steady state performances, under sinusoidal excitation, are needed. An interesting alternative is to use a fixed-mesh method of movement simulation [3]. This technique doesn't consider the teeth permeance variation due to the movement. Nevertheless, it gives very good results in steady state while reducing the cpu time compared to a moving-mesh technique to account for movement.

To drastically reduce the cpu time, time-harmonic (TH) techniques are more attractive to compute steady state performances of induction machines (IM) under sinusoidal excitation. They are strictly valid in the linear case although the saturation can be considered in an average sense. In [4] this method is used but the induced currents due to the air-gap spatial harmonics are not properly considered. In [5]-[6], coupling schemes of the magnetic field in the air-gap are proposed to convert the space harmonics at the right frequencies.

All these TH techniques have been widely applied to squirrel cage IM. Surprisingly, very few works are dedicated to

Manuscript received July 6, 2015. Corresponding author: S. Mezani (email: smail.mezani@univ-lorraine.fr).

Color versions of one or more of the figures in this paper are available online at http://ieeexplore.ieee.org.

Digital Object Identifier (inserted by IEEE)
WRIM computation using TH methods.

We propose in this paper an approach based on FE-circuit analysis that allows a fast and precise computation of WRIM performances in steady state. The magnetic field is truncated so only the principal air-gap space harmonic is considered. A similar approach has been successfully used for the computation of squirrel-cage induction motors [6]-[7]. A FE computation is needed for each value of the slip frequency in the rotor bars. In this paper, it will be shown that only four FE magnetostatic computations are necessary to determine the WRIM performances.

\section{II.THE ELECTROMAGNETIC MODEL}

A magnetic vector potential (MVP) formulation is used under the usual plane 2D approximation. The background of the electromagnetic model is the same as the one described in [6][7] for squirrel cage induction motors. The machine is split into two domains of resolution noted $D_{s}$ and $D_{r}$, Fig.1. Both domains include the air-gap domain $D_{g}$, so this method has been called the "double air-gap method". The ferromagnetic materials are considered linear in this study.

\section{A. The double air-gap method applied to WRIM}

The principle of the double air-gap method applied to WRIM is presented here in a technical way. A deep mathematical formulation can be found in [7] for squirrel-cage IM.

A WRIM usually consists of two p pole-pair 3-phase windings in the stator and the rotor armatures. The rotor winding is short-circuited at normal operation. In steady state operation, the stator winding is supplied with a voltage source at a time pulsation $\omega_{s}$. For a rotor speed $\Omega$, the time pulsations induced in the rotor winding is $\omega_{r}$. The slip is then defined as

$s=\left(\omega_{s}-\mathrm{p} \Omega\right) / \omega_{s}=\omega_{r} / \omega_{s}$

We consider that only the first space harmonic of $p$ polepair exists in the air gap. In $D_{s}$ and $D_{r}$, the MVP has two determinations expressed as follows 


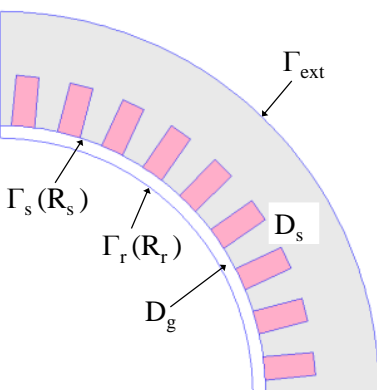

(a)

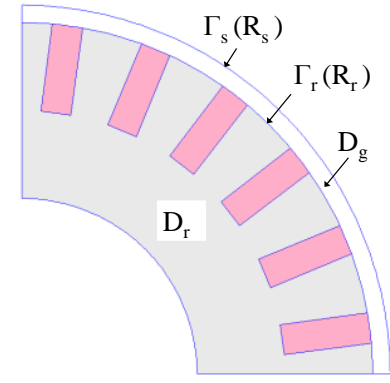

(b)
Fig. 1. Stator (a) and rotor (b) domains

$a_{s}(P, t)$

$=\sqrt{2} \operatorname{Re}\left[\left(\overline{I_{s}} \cdot \overline{X_{s}}(P)+\overline{C_{s}} \cdot \overline{A_{s}}(P)\right) \exp \left(j \omega_{s} t\right)\right]$ in $D_{s}$

$a_{r}\left(P^{\prime}, t\right)$

$=\sqrt{2} \operatorname{Re}\left[\left(\overline{I_{r}} \cdot \overline{X_{r}}\left(P^{\prime}\right)+\overline{C_{r}} \cdot \overline{A_{r}}\left(P^{\prime}\right)\right) \exp \left(j s \omega_{s} t\right)\right]$ in $D_{r}$

Re stands for "real part of". The points $\mathrm{P}$ and $\mathrm{P}$ ' have the coordinates $(\mathrm{r}, \theta)$ in $\mathrm{D}_{\mathrm{s}}$ and $\left(\mathrm{r}, \theta^{\prime}\right)$ in $\mathrm{D}_{\mathrm{r}}$ respectively.

$\overline{X_{s}}$ and $\overline{X_{r}}$ are complex valued MVPs due to unity stator $\left(\overline{I_{s}}\right)$ and rotor $\left(\overline{I_{r}}\right)$ currents respectively. $\overline{A_{s}}$ and $\overline{A_{r}}$ are unity MVPs due to rotor and stator reaction fields respectively. $\overline{C_{s}}$ and $\overline{C_{r}}$ are phasors.

Furthermore, in addition to (2) and (3), $a_{s}$ and $a_{r}$ must coincide everywhere in the air-gap. To do so, it is sufficient to ensure the following continuity relations in $D_{g}$

$\begin{cases}a_{s}\left(R_{s}, \theta, t\right)=a_{r}\left(R_{s}, \theta^{\prime}, t\right) & \text { on } \Gamma_{s} \\ a_{s}\left(R_{r}, \theta, t\right)=a_{r}\left(R_{r}, \theta^{\prime}, t\right) & \text { on } \Gamma_{r}\end{cases}$

Indeed, $a_{s}$ and $a_{r}$ are harmonic functions in the air-gap (they are solution of the Laplace equation) so their equality on the air-gap boundaries $\Gamma_{s}$ and $\Gamma_{r}$ implies their coincidence everywhere in the air-gap.

In order to determine the MVP everywhere in the machine using (2)-(3), we need to compute $\overline{C_{s}}, \overline{C_{r}}$ as well as $\overline{I_{s}}, \overline{I_{r}}$ (if the machine is supplied from a voltage source). We also need to determine the elementary MVPs $\overline{A_{s}}, \overline{X_{s}}, \overline{A_{r}}$ and $\overline{X_{r}}$.

\section{B. Computation of $\overline{X_{s}}$ and $\overline{A_{s}}$ in $D_{s}$}

$\overline{X_{\mathrm{s}}}$ corresponds to the source problem. The stator windings are supplied by a unity 3 -phase current such as

$\overline{I_{s, k}}=\exp ((k-1) j 2 \pi / 3), \quad k=1,2,3$

where $\mathrm{k}$ is the phase index. We set $\overline{X_{\mathrm{s}}}=0$ on $\Gamma_{\text {ext }}$.

On $\Gamma_{r}$, we can either set $\overline{X_{\mathrm{s}}}=0$ or $\frac{\partial \overline{X_{\mathrm{s}}}}{\partial r}=0$.

We solve by FE the Laplace (in the iron parts and the air-gap) and Poisson (in the slots) partial differential equations (PDEs). Then we compute the $\mathrm{p}^{\text {th }}$ harmonic Fourier coefficient noted $\overline{\mu_{s s}}$ on $\Gamma_{s}$ and $\overline{\mu_{s r}}$ on $\Gamma_{r}$.

We also compute the phase magnetic flux linkage noted $\overline{\varphi_{\mathrm{sX}}}$ (the choice of the phase is arbitrary).
$\overline{A_{\mathrm{s}}}$ corresponds to the rotor armature reaction. The 3-phase stator windings are not supplied and we set $\overline{A_{\mathrm{s}}}=0$ on $\Gamma_{\text {ext }}$.

On $\Gamma_{r}$, we can either set $\overline{A_{\mathrm{s}}}=\exp (j p \theta)$ or $\frac{\partial \overline{A_{s}}}{\partial r}=\exp (j p \theta)$.

We solve by FE the Laplace PDE (iron parts, slots and airgap). Then we compute the $\mathrm{p}^{\text {th }}$ harmonic Fourier coefficients noted $\overline{\lambda_{s s}}$ on $\Gamma_{s}$ and $\overline{\lambda_{s r}}$ on $\Gamma_{r}$.

We also compute the phase magnetic flux linkage noted $\overline{\varphi_{\mathrm{sA}}}$.

C. Computation of $\overline{X_{r}}$ and $\overline{A_{r}}$ in $D_{r}$

As for the stator domain, the same computations are performed in the rotor domain. The boundary conditions are set on $\Gamma_{s}$.

The solution of the rotor source problem $\overline{X_{r}}$ allows to compute the $\mathrm{p}^{\text {th }}$ harmonic Fourier coefficients noted $\overline{\mu_{r s}}$ on $\Gamma_{s}$ and $\overline{\mu_{r r}}$ on $\Gamma_{r}$.

We also compute the phase magnetic flux linkage noted $\overline{\varphi_{\mathrm{rX}}}$.

The MVP $\overline{A_{\mathrm{r}}}$ corresponds to the stator armature reaction. Its computation allows the determination of the $\mathrm{p}^{\text {th }}$ harmonic Fourier coefficients noted $\overline{\lambda_{r s}}$ on $\Gamma_{s}$ and $\overline{\lambda_{r r}}$ on $\Gamma_{r}$.

We also compute the phase magnetic flux linkage noted $\overline{\varphi_{\mathrm{rA}}}$.

With the boundary conditions on $\Gamma_{s}$ and $\Gamma_{r}$ (2 possibilities for each elementary problem), there will be 8 combinations for the $4 \mathrm{FE}$ computations. This allow to adapt the first harmonic approximation to the physical situation of a given problem. A discussion on the subject can be found in [7].

\section{Determination of $\overline{C_{s}}, \overline{C_{r}}, \overline{I_{s}}$ and $\overline{I_{r}}$}

In order to determine $\overline{C_{s}}, \overline{C_{r}}, \overline{I_{s}}$ and $\overline{I_{r}}$, we need a set of 4 algebraic equations. Two of them come from the equalities given in (4) and the two others are related to the per phase circuit equation of the stator and the rotor.

Using (4), one can write

$\overline{\lambda_{s s}} \cdot \overline{C_{s}}+\overline{\mu_{s s}} \cdot \overline{I_{s}}=\overline{\lambda_{r s}} \cdot \overline{C_{r}}+\overline{\mu_{r s}} \cdot \overline{I_{r}}$ on $\Gamma_{s}$
$\overline{\lambda_{s r}} \cdot \overline{C_{s}}+\overline{\mu_{s r}} \cdot \overline{I_{s}}=\overline{\lambda_{r r}} \cdot \overline{C_{r}}+\overline{\mu_{r r}} \cdot \overline{I_{r}}$ on $\Gamma_{r}$

The stator per phase circuit equation is

$\overline{V_{s}}=r_{s} \overline{I_{s}}+j \omega_{s} l_{s e w} \overline{I_{s}}+j \omega_{s}\left(\overline{I_{s}} \cdot \overline{\varphi_{s X}}+\overline{C_{s}} \cdot \overline{\varphi_{s A}}\right)$

and the rotor per phase circuit equation is

$0=r_{r} / s \cdot \overline{I_{r}}+j \omega_{s} l_{r e w} \overline{I_{r}}+j \omega_{s}\left(\overline{I_{r}} \cdot \overline{\varphi_{r X}}+\overline{C_{r}} \cdot \overline{\varphi_{r A}}\right)$

$\bar{V}_{S}$ is the stator phase voltage,

$r_{s}$ and $r_{r}$ are the stator and rotor phase resistances respectively, $l_{\text {sew }}$ and $l_{\text {rew }}$ are the stator and rotor phase end-winding leakage inductances respectively. 
The relations (6)-(9) allow the determination of the unknowns of the problem. It is clear that only 4 FE complexmagnetostatic computations are required to have the solution for any slip value (the slip only appears in the rotor circuit equation (9)).

\section{APPLICATION EXAMPLE}

The proposed method has been tested on a short-circuited rotor WRIM rated at $100 \mathrm{~kW}, 980 \mathrm{rpm}(\mathrm{s}=2 \%)$. The main parameters of the machine are given in Table I. This is an inverse-type WRIM having an external rotor used in a novel topology of a magnetically geared induction machine (MaGIM) [8].

As stated above, the boundary condition for each elementary problem use the MVP or its derivative with respect to $r$. It is worth to note that at low slip values $(\mathrm{s}<0.1)$, the same results are obtained whether the used boundary condition. However, compared to a time-stepping computation, the best results are obtained when using, as a boundary condition, the MVP derivative for the source problems and the MVP for the reaction problems. The following results are then obtained for this last combination.

Fig. 2 presents the flux lines in the stator and the rotor at rated slip $(s=0.02)$. In order to appreciate the effectiveness of the coupling scheme, the harmonic spectra of the radial flux density distribution along a circular path in the middle of the air gap is shown in Fig. 3. It can be seen that the rms values of the fundamental harmonic computed in $D_{s}$ and $D_{r}$ are very close, they are also very similar to the one obtained from a time-stepping simulation $(0.5 \mathrm{~T}$ for our method and $0.515 \mathrm{~T}$ for the time-stepping computation).

The performances of the studied WRIM at rated speed are given in Table II. It can be seen that the results computed using the proposed method are consistent with those obtained from conventional full time-harmonic and time stepping models of the machine. Furthermore, one can note that the machine is almost unsaturated. Indeed, medium to high power WRIM are usually designed to operate at low saturation level [1], so there is clearly a place for using a linear model for such type of machine.

Fig.4. shows the computed electromagnetic torque and per phase rms stator current for slip values ranging from 0 to 0.3 . It can be seen that the obtained results using our model are in good agreement with those obtained using conventional timeharmonic and time-stepping FE models. The maximal error is about $10 \%$ for the torque and less than $2 \%$ for the current.

The overall computation time is about $6 \mathrm{~s}$ using the proposed method (4 FE computations). For the full time-harmonic model, the computation time for 30 slip values is about 70 s. Each time-stepping computation last several minutes to reach steady state.

Another remark can also be made. The computation time of our method is comparable to the one which one obtains from a usual equivalent circuit analysis. Indeed, the identification of the parameters of the equivalent circuit requires two FE com- putations for the whole machine geometry (no-load and locked rotor operation). The cpu time for these 2 computations is almost the same as for the $4 \mathrm{FE}$ computations of half a machine ( 2 stator and 2 rotor FE computations).

TABLE I

MAIN PARAMETERS OF THE WRIM

\begin{tabular}{ll}
\hline \hline & Value \\
Parameter description & $100 \mathrm{~kW}$ \\
\hline Rated power & $400 \mathrm{~V}(\Delta$ connection) \\
Stator rated voltage, Vs & $50 \mathrm{~Hz}$ \\
Stator frequency & 3 \\
Pole pair, p & $980 \mathrm{rpm}$ \\
Rated speed, $\Omega$ & $72(10 / 12$ shortening) \\
Number of stator slots & $54(7 / 9 \mathrm{shortening}, \mathrm{Y})$ \\
Number of rotor slots & $201.4 \mathrm{~mm}$ \\
Stator outer radius & $0.6 \mathrm{~mm}$ \\
Air gap & $260 \mathrm{~mm}$ \\
Active length & $49 \mathrm{~mm}$ \\
Stator slot height & $2.2 \mathrm{~mm}$ \\
Stator slot isthmus widh & $8.6 \mathrm{~mm}$ \\
Stator tooth width (rectangular) & $42 \mathrm{~mm}$ \\
Stator back-iron height & $32 \mathrm{~mm}$ \\
Rotor slot height & $2.6 \mathrm{~mm}$ \\
Rotor slot isthmus width & $0.5 \mathrm{~mm}$ \\
Slot isthmus height (stator and rotor) & $10.9 \mathrm{~mm}$ \\
Rotor tooth width (rectangular) & $50 \mathrm{~mm}$ \\
Rotor back-iron height & 72 \\
Turns in series per phase (stator and rotor) & $89 \mathrm{~m} \Omega(78 \mathrm{~m} \Omega)$ \\
Stator $r_{\mathrm{s}}$ (Rotor $\mathrm{r}_{\mathrm{r}}$ ) per phase resistance & $0.27 \mathrm{mH}(0.25 \mathrm{mH})$ \\
Stator (Rotor) end-winding leakage & \\
inductance & \\
\hline \hline
\end{tabular}

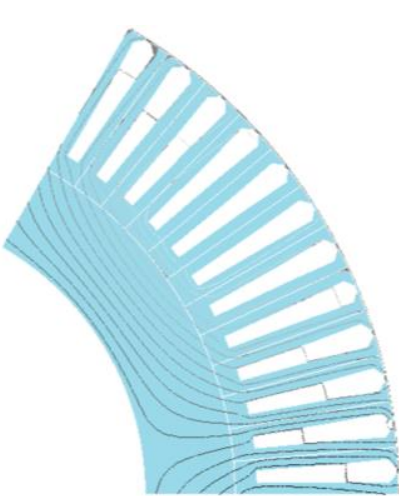

(a)

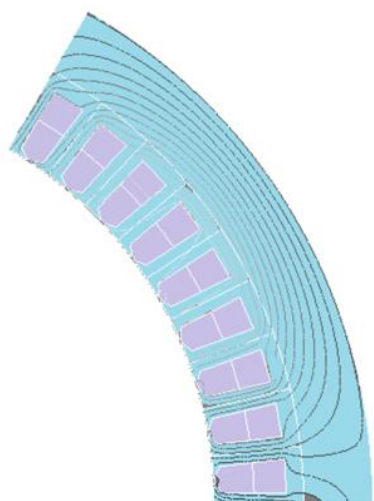

(b)
Fig.2. Magnetic flux lines (a) in $D_{s}$, (b) in $D_{r}$ at rated slip $s=0.02$

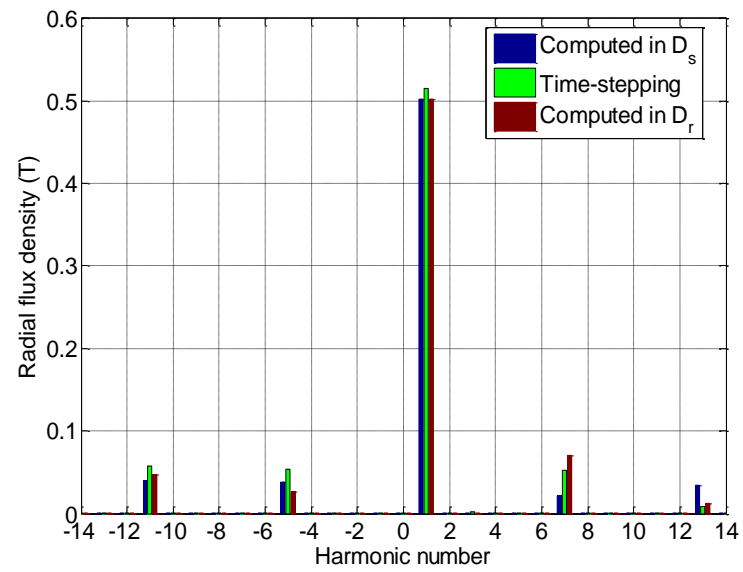

Fig.3. Harmonic spectra of the radial flux density in the middle of the air gap 
TABLE II

Main performances of the $100 \mathrm{~kW}$ WRIM at rated operation, $\mathrm{s}=0.02$

\begin{tabular}{lllll}
\hline \hline Quantity & $\begin{array}{l}\text { Proposed } \\
\text { method }\end{array}$ & $\begin{array}{l}\text { Time } \\
\text { harmonic }\end{array}$ & $\begin{array}{l}\text { Time } \\
\text { stepping } \\
\text { (linear) }\end{array}$ & $\begin{array}{l}\text { Time } \\
\text { stepping } \\
\text { (saturated) }\end{array}$ \\
\hline $\begin{array}{l}\text { Stator current, } \\
\text { A rms }\end{array}$ & 95 & 95.7 & 94.1 & 96 \\
$\begin{array}{l}\text { Torque, Nm } \\
\text { Power factor }\end{array}$ & 930 & 958 & 975 & 985 \\
\hline \hline
\end{tabular}

WRIM can develop high starting torque with reasonably low current by inserting external resistances $R_{\text {ext }}$ in the rotor circuit. Fig. 5 presents the torque and the absorbed current of the studied WRIM at standstill $(\mathrm{s}=1)$. Again, the agreement between the results issued from our model and those of the time-harmonic computation is good (about 10\% maximal error for the torque evaluation and $2 \%$ for the current). For $R_{\text {ext }}=0.8$ $\Omega$, the starting torque can reach more than $2 \mathrm{kNm}$ (twice the rated value) while the starting current is about $280 \mathrm{~A}(2.8$ times the rated current).

\section{CONCLUSION}

The presented method, based on the first space harmonic approximation and coupled FE-circuit equation of WRIM, is very fast and sufficiently accurate compared to conventional time-harmonic and time-stepping methods. As an extension of this work, the consideration of higher space harmonics and the magnetic saturation in our model will result in very powerful tool for WRIM analysis. In future works, the proposed method will be used to analytically compute wound rotor induction motors using the subdomain method.

\section{V.ACKNOWLEDGEMENT}

This work is undertaken under an EU Marie-Curie IEF project MaGIM, No PIEF-GA-2012-330833

\section{REFERENCES}

[1] I. Boldea, and S. A. Nasar, The Induction Machines Design Handbook, $2^{\text {nd }}$ ed., CRC Press, 2009, $845 \mathrm{p}$.

[2] A. C. Smith, S. Williamson, and J. R. Smith, "Transient currents and torques in wound-rotor induction motors using the finite-element method," IEE Proc., Vol. 137, Pt. B, No. 3, pp. 160-173, May 1990.

[3] A. Demenko and I. Nowak, "Finite element analysis of saturation effects in a squirrel cage electrical machine," COMPEL, Vol. 15, no. 4, pp. 8895, 1996.

[4] E. Vassent, G. Meunier, and J. C. Sabonnadière, "Simulation of induction machine operation using complex magnetodynamic finite elements," IEEE Trans. Magn., vol. 25, pp. 3064-3066, Jul. 1989.

[5] H. De Gersem and K. Hameyer, "Air-Gap Flux Splitting for the TimeHarmonic Finite-Element Simulation of Single-Phase Induction Machine," IEEE Trans. Magn., vol. 38, no. 3, pp. 1221-1223, Mar. 2002.

[6] G. Vinsard, and B. Laporte, "An analysis of the first harmonic method to compute induction motors," IEEE Trans. Magn., vol. 31, no. 3, pp. 2162-2165, May 1995.

[7] S. Mezani, N. Takorabet, and B. Laporte, "Saturation and Space Harmonics in the Complex Finite Element Computation of Induction Motors, " IEEE Trans. Magn., vol. 41, no. 5, pp. 1460-1463, May 2005.

[8] S. Mezani, T. Hamiti, L. Belguerras, T. Lubin, M. Rashed, C. Gerada, "Magnetically Geared Induction Machines," IEEE Trans. Magn., Early access paper, DOI:10.1109/ TMAG.2015.2443252.

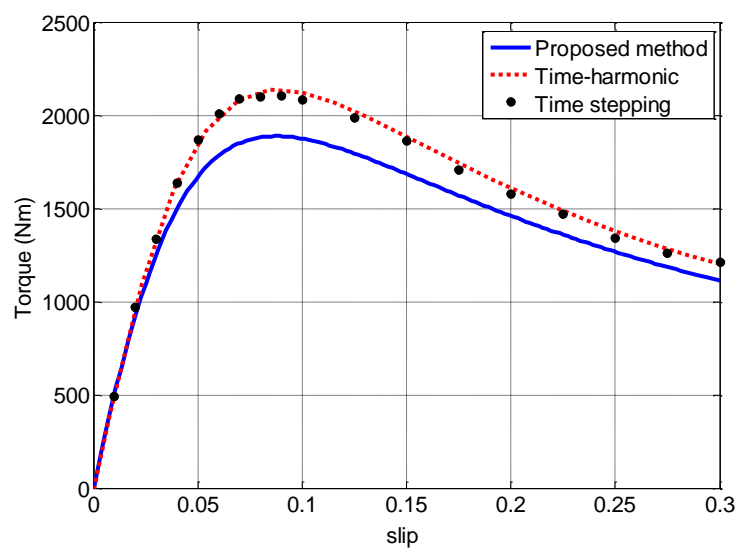

(a)

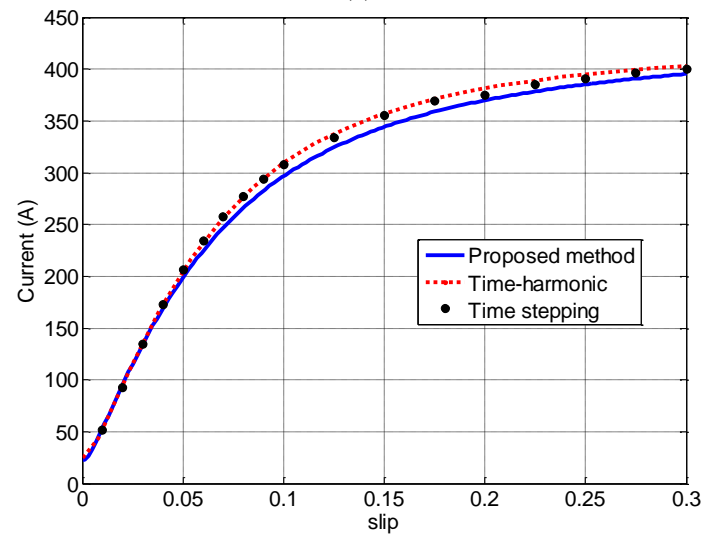

(b)

Fig. 4. Torque (a) and rms stator current (b) vs. slip curves

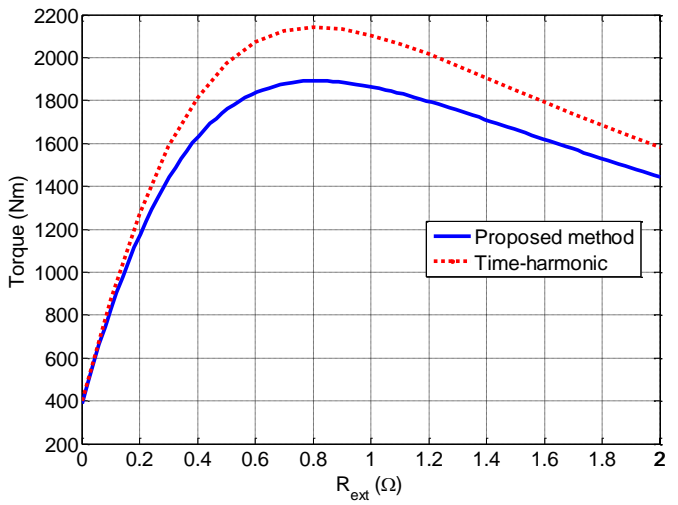

(a)

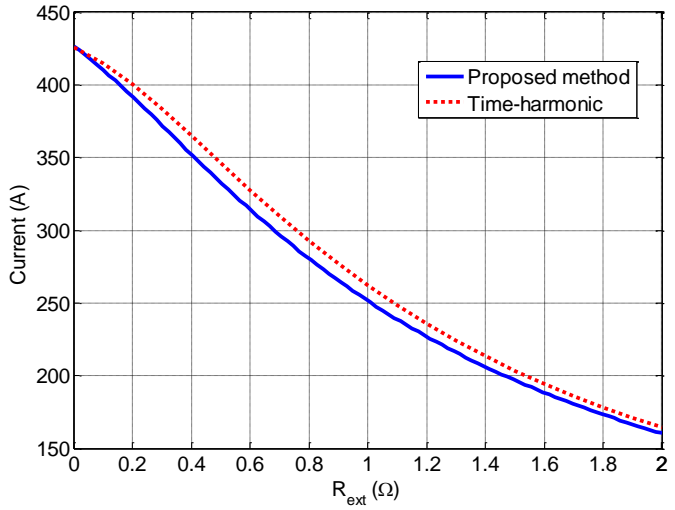

(b)

Fig. 5. Torque (a) and rms stator current (b) vs. $R_{\text {ext }}$ at standstill 